\title{
Early myelin breakdown following sural nerve crush: a freeze-fracture study
}

A.M.B. Martinez and S. Canavarro
Departamento de Histologia e Embriologia, Instituto de Ciências Biomédicas, Universidade Federal do Rio de Janeiro, Rio de Janeiro, RJ, Brasil

\section{Correspondence \\ A.M.B. Martinez \\ Departamento de Histologia e \\ Embriologia, Bloco F, ICB, UFRJ \\ Av. Brigadeiro Trompowski, s/n \\ 21941-590 Rio de Janeiro, RJ \\ Brasil \\ E-mail: martinez@ chagas.biof.ufrj.br}

Research supported by CAPES, CNPq, FAPERJ and FUJB.

Received April 5, 2000

Accepted September 13, 2000

\section{Abstract}

In this study we describe the early changes of the myelin sheath following surgical nerve crush. We used the freeze-fracture technique to better evaluate myelin alterations during an early stage of Wallerian degeneration. Rat sural nerves were experimentally crushed and animals were sacrificed by transcardiac perfusion $30 \mathrm{~h}$ after surgery. Segments of the nerves were processed for routine transmission electron microscopy and freeze-fracture techniques. Our results show that $30 \mathrm{~h}$ after the lesion there was asynchrony in the pattern of Wallerian degeneration, with different nerve fibers exhibiting variable degrees of axon disruption. This was observed by both techniques. Careful examination of several replicas revealed early changes in myelin membranes represented by vacuolization and splitting of consecutive lamellae, rearrangement of intramembranous particles and disappearance of paranodal transverse bands associated or not with retraction of paranodal myelin terminal loops from the axolemma. These alterations are compatible with a direct injury to the myelin sheath following nerve crush. The results are discussed in terms of a similar mechanism underlying both axon and myelin breakdown.

\section{Key words}

- Myelin breakdown

- Wallerian degeneration

- Freeze-fracture

- Crush lesion

- Sural nerve
It is generally accepted that axonal cytoskeleton breakdown during Wallerian degeneration precedes myelin disruption (for a review, see Ref. 1). However, the early changes in myelin sheath following nerve injury are not well understood. We have sometimes observed in fibers undergoing early Wallerian degeneration areas of myelin disruption in the presence of a normal or partially disrupted axoplasm content (data not shown). This finding has also been reported by others (2,3). Ludwin (2) described simultaneous degeneration of the axon and myelin after transection of the optic nerve. It is, however, very common to interpret these findings as artifacts due to poor fixation. If this is not true, it is possible that myelin is directly affected by nerve injury.

Understanding the mechanism of myelin breakdown after nerve crush might be useful to elucidate the pathogenesis of myelin dissolution that occurs in Wallerian degeneration and many demyelinating pathologies which are associated with central and peripheral nervous system diseases. We therefore decided to better evaluate this issue by means of a technique that reveals the internal organization of membranes. Thus, in the present study we investigated the early myelin alterations which follow surgical nerve crush 
using the freeze-fracture technique. Thirty hours after crush was the time chosen for the study because there are fibers in different stages of Wallerian degeneration. At that time, approximately $25 \%$ of fibers no longer exhibit cytoskeletal components but the remaining $75 \%$ are either normal or display partial disruption of the axoplasmic cytoskeleton (4).

Ten Wistar rats were anesthetized and their right sural nerves crushed using a fine forceps and a moderate pressure for $10 \mathrm{~s}$. Left sural nerves were used as controls. Thirty hours after the crush the animals were perfused intracardially with $2 \%$ glutaraldehyde and $2 \%$ paraformaldehyde in $0.1 \mathrm{M}$ phos-

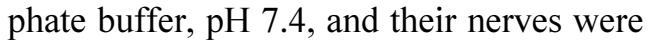
dissected out and processed for freeze-fracture. Briefly, fragments of the nerve's distal stump (the immediately distal $0.5 \mathrm{~cm}$ segment was discarded to avoid the traumatic area) were infiltrated in glycerol, mounted on specimen support disks, frozen in liquid nitrogen and fractured at $-196^{\circ} \mathrm{C}$ in Balzers freeze-fracture equipment, followed by platinum shadowing at a $45^{\circ}$ angle. Replicas were obtained after sodium hypochloride digestion, collected on copper grids and observed under a Zeiss 900 transmission electron microscope. We also processed tissue for conventional transmission electron microscopy. Briefly, fixed segments of the nerve's distal stump were post-fixed in $1 \%$ osmium tetroxide, dehydrated in acetone, embedded in PolyBed resin, cut on an RMC ultramicrotome and observed under a Zeiss transmission electron microscope.

Analysis of ultrathin sections revealed myelinated nerve fibers of normal appearance in controls (Figure 1A). In degenerating nerves we could observe normal looking myelinated nerve fibers together with fibers exhibiting partial or total granular dissolution of the axoplasm (Figure 1B).

Careful examination of several replicas revealed myelinated nerve fibers fractured at different angles exhibiting several aspects of normal and degenerating nerve fibers. In control nerves it was possible to visualize myelinated nerve fibers displaying a normal axoplasm with its typical cytoskeleton and organelle content surrounded by a compact myelin sheath. In Figure 1C we can observe a myelinated nerve fiber obliquely fractured exhibiting the P face of the outer Schwann cell membrane with its outer mesaxon, Schwann cell cytoplasm, several consecutive layers of myelin and the axoplasm with particles belonging to proteins of the cytoskeleton. At a higher magnification we can observe in another fiber the $\mathrm{P}$ face of a myelin lamella with homogeneously distributed intramembranous particles (Figure 1D). This observation was a common finding in all replicas from normal control nerves. At $30 \mathrm{~h}$ after the crush we could observe fibers which were either normal or exhibited different stages of degeneration. So, it was possible to visualize fibers in which myelin and axoplasm were apparently normal similar to those belonging to control nerves. However, in some fibers there were more obvious alterations in myelin structure represented by areas in which the normal compact appearance was lost. Figure 2A and B represents two such areas. Here (Figure 2A) one myelin lamella was longitudinally fractured exhibiting a $\mathrm{P}$ face in which the normal aspect was replaced with areas resembling "blebs" that are apparently splitting consecutive myelin lamellae. These regions could represent areas of early myelin vacuolization and dissolution. In these areas no intramembranous particles were observed. Also, several replicas of degenerating nerve fibers exhibited longitudinally fractured myelin lamellae showing depressed regions in which the intramembranous particles had completely disappeared (Figure 2B). These depressions were surrounded by areas which remained apparently normal even though the intramembranous particles appeared to be more densely packed, suggesting a redistribution of such particles. Taken together, these 
data indicate that myelin alterations can appear at a very early stage of Wallerian degeneration and are characterized by vacuolization of myelin lamellae and separation and rearrangement of intramembranous particles. Another interesting finding was the pres- ence, in one replica, of a paranode (Figure 2C) which exhibited the $P$ face of the axolemma with its indentations formed by myelin terminal loops (MTL) which appeared as parallel transverse bands running perpendicularly to the long axis of the axon. The
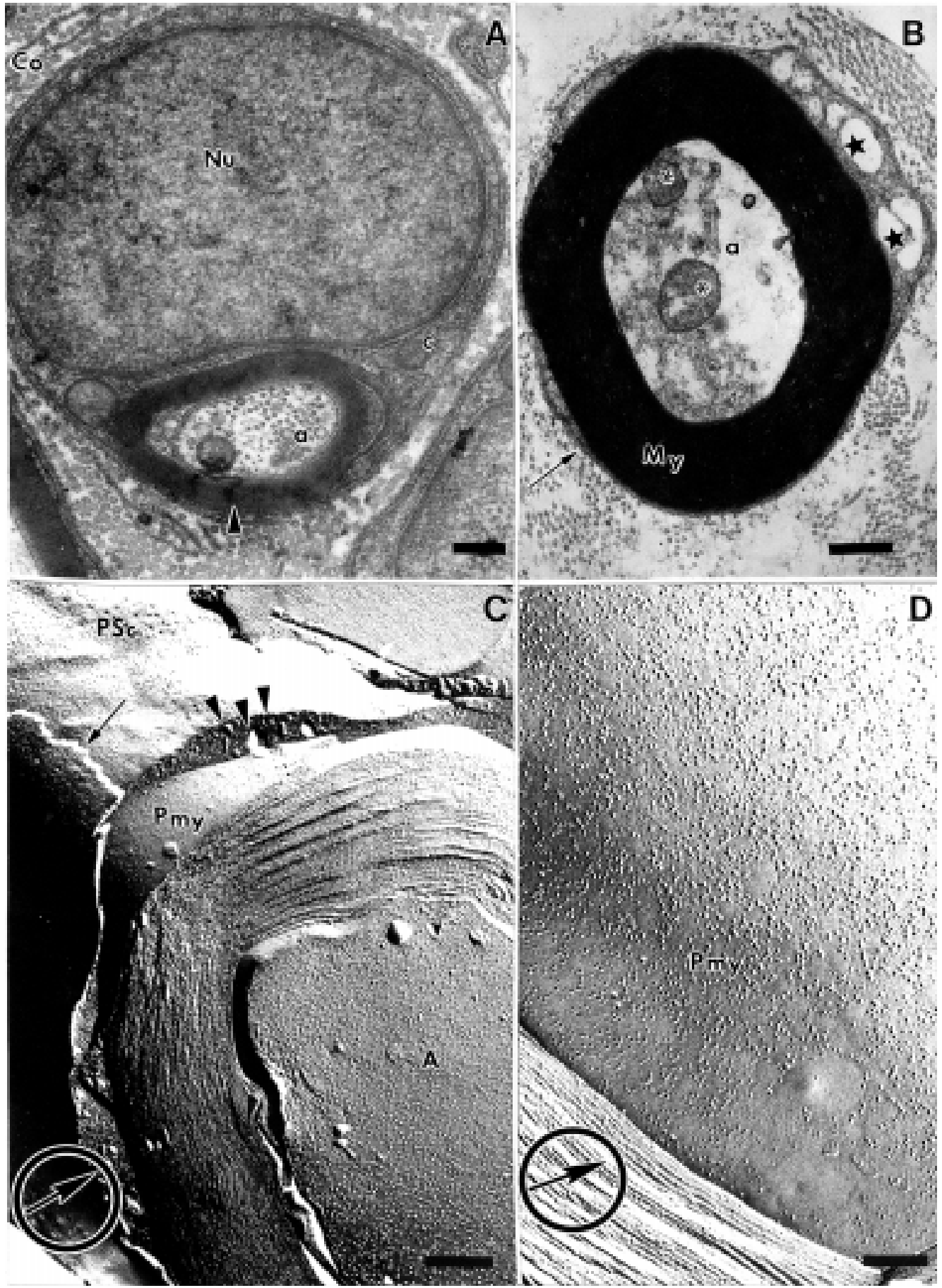

Figure 1 - Transmission electron micrographs of normal and degenerating myelinated nerve fibers. A, Normal myelinated nerve fiber showing typical axoplasm content (a), compact myelin lamellae (arrowhead), Schwann cell cytoplasm (c), nucleus $(\mathrm{Nu})$ and collagen fibrils (Co). B, Degenerating nerve fiber showing an almost complete disintegration of axoplasm (a), apparently normal myelin sheath (My) and accumulation of organelles (asterisks). Observe enlarged cisternae of rough endoplasmic reticulum (stars) and the presence of an intact basal lamina (arrow). C, Replica of a myelinated nerve fiber exhibiting the $P$ face of the outer Schwann cell membrane (PSc) and the $P$ face of a myelin lamella (Pmy). The arrow points to mesaxon ridge and arrowheads to the cytoplasm of Schwann cells. A, Axon. D, Replica of a myelinated nerve fiber showing the $P$ face of a myelin lamella (Pmy) with randomly distributed intramembranous particles. Encircled arrows in the lower left comers of Figure $1 \mathrm{C}$ and $D$ indicate the direction of platinum shadowing. Bars: $A, D$ $=0.4 \mu \mathrm{m} ; \mathrm{B}, \mathrm{C}=0.6 \mu \mathrm{m}$. 
Figure 2 - Freeze-fracture replicas of degenerating myelinated nerve fibers. A, Replica of a degenerating nerve fiber showing vacuolization of myelin (arrows). Observe that the remaining myelin lamellae (My) are still compact. B, Replica of a myelinated nerve fiber displaying $P$ faces of myelin lamellae in which we can observe particle-free depressed regions (asterisks) surrounded by areas showing densely packed intramembranous particles. The arrow points to obliquely fractured myelin lamellae. C, P face of a paranodal axolemma showing identations (thin arrows) produced by myelin terminal loops (blank arrows). Observe that the myelin terminal loop closest to the nodal area is retracted from the axolemma (arrowhead). Black arrows point to 2 next myelin terminal loops which remain attached to the axolemma. Note that the corresponding bands are missing. The remaining myelin terminal loops (blank arrows) and bands still show a normal appearance. Bars: $A, C=0.6 \mu \mathrm{m} ; \mathrm{B}=1.1 \mu \mathrm{m}$. majority of these bands and MTL appeared normal but, as can be observed in Figure 2C, the first paranodal MTL (closest to the node) is detached from the axolemma and the associated transverse band is missing. Interestingly, the next two MTL are apparently still
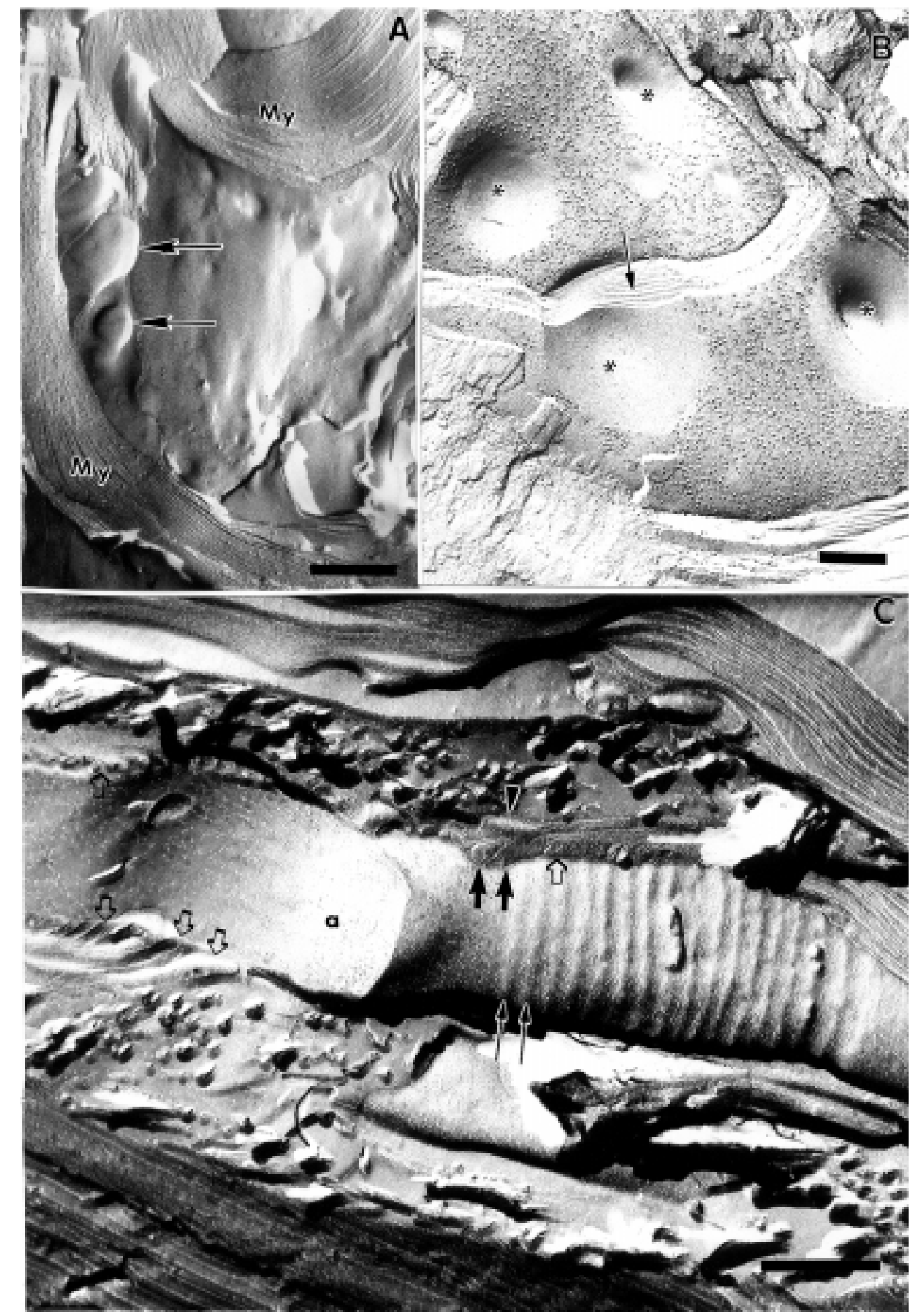
attached to the axolemma but their corresponding bands have disappeared. However, as can be easily observed in Figure $2 \mathrm{C}$, the intramembranous particles associated with the transverse bands are still present. This data suggest that transverse bands disappear before MTL retraction and that this event is not a consequence of the latter. In agreement with these findings, in a previous study we showed that at $30 \mathrm{~h}$ after crush many nodes exhibit an increase in the nodal gap but labeling for sodium channel proteins is still positive (5), indicating that MTL retraction is an early event and might occur before the intramembranous particles degenerate and disappear. Previous works have also shown early myelin detachment from the axolemma (6), but Abrahams et al. (7), who also addressed this issue, did not find this alteration at $24 \mathrm{~h}$ after nerve crush. Therefore, we suggest that $30 \mathrm{~h}$ is a critical period for the initiation of myelin retraction.

Our results present evidence that in the course of early Wallerian degeneration the myelin sheath suffers alterations in its structure which are represented by splitting and vacuolization of consecutive lamellae, redistribution of intramembranous particles and disappearance of paranodal parallel transverse bands which can appear associated or not with retraction of MTL from the paranodal axolemma.

The mechanism underlying the initiation of axon degeneration has been a subject of interest for many researchers and, although it is not completely elucidated, many of its aspects have been clarified recently. It has been shown that axonal cytoskeleton degeneration occurs due to an increase in intra- axonal calcium concentration (8-16) which activates proteases such as calpains (17). These enzymes are normally present inside axons and are only activated when there is an increase in intra-axonal calcium concentration. Calpains have also been found inside astrocytes and Schwann cell cytoplasm during Wallerian degeneration (2) and have also been implicated in the degradation of myelin basic protein which is a normal component of the myelin sheath in central and peripheral myelinated nerve fibers (2). Therefore, it is possible that Schwann cell calpains are also activated by an increase in calcium ions inside the cytoplasm as a general tissue response to mechanical trauma (nerve crush) and consequently could be responsible for the early myelin alterations as observed in our study and others on nerve degeneration. The involvement of proteolytic enzymes in the mechanism of myelin breakdown has also been described in the course of demyelinating diseases (18).

The route of calcium entry into the axon is currently being studied in many research laboratories. Recently it has been postulated that the mechanism of calcium entry is probably due to a reverse operation of the $\mathrm{Na}^{+}-$ $\mathrm{Ca}^{+}$exchanger $(19,20)$ which pumps calcium into the axon as a response to ATP deprivation and failure of $\mathrm{Na}^{+}-\mathrm{K}^{+}$ATPase. Although this putative route was described as the mechanism of calcium entry into the axons it is very likely that it can also underlie the mechanism of calcium entry into Schwann cells and therefore be responsible for calpain activation and myelin degradation at an early stage of nerve degeneration.

\section{References}

1. J oseph BS (1973). Somatofugal events in Wallerian degeneration: a conceptual overview. Brain Research, 59: 1-18.

2. Ludwin SK (1995). The axon. In: Waxman SG, Kocsis J D \& Stys PK (Editors), Pathology of the Myelin Sheath. Oxford Univer- sity Press, Oxford.

3. Cullen MJ (1988). Freeze-fracture analysis of myelin membrane changes in Wallerian degeneration. J ournal of Neurocytology, 17: 105-115.

4. Malbouisson AMB, Ghabriel MN \& Allt G
(1984). The non-directional pattern of axonal changes in Wallerian degeneration. A computer-aided morphometric analysis. J ournal of Anatomy, 139: 159-174.

5. Martinez AMB (1999). Distribution of sodium and potassium channels as well as 
myelin associated glycoprotein (MAG) during the early stages of Wallerian degeneration. J ournal of Submicroscopic Cytology and Pathology, 31: 73-81.

6. Ishise J \& Rosenbluth J (1987). Nodal and paranodal structure during Wallerian degeneration in frog spinal nerve. Brain Research, 418: 85-97.

7. Abrahams PH, Day A \& Allt G (1981). The node of Ranvier in early Wallerian degeneration: A freeze-fracture study. Acta Neuropathologica, 54: 95-100.

8. Schlaepfer WW (1971). Experimental alterations of neurofilaments and neurotubules by calcium and other ions. Experimental Cell Research, 67: 73-80.

9. Schlaepfer WW (1974). Calcium-induced degeneration of axoplasm in isolated segments of rat peripheral nerve. Brain Research, 69: 203-215.

10. Schlaepfer WW (1977). Structural alterations of peripheral nerve induced by the calcium ionophore A23187. Brain Research, 136: 1-9.
11. Schlaepfer WW \& Hasler MB (1979). Characterization of the calcium-induced disruption of neurofilaments in rat peripheral nerve. Brain Research, 168: 299-309.

12. Kamakura K, Ushiura S, Sugita $H$ \& Toyokuura Y (1983). Identification of $\mathrm{Ca}^{2+}$ activated neutral protease in the peripheral nerves. J ournal of Neurochemistry, 40: 908-913.

13. Mata M, Staple J \& Fink DJ (1986). Changes in intra-axonal calcium distribution following nerve crush. J oumal of Neurobiology, 17: 449-467.

14. Glass J D, Schryer BL \& Griffin J W (1994). Calcium-mediated degeneration of the axonal cytoskeleton in the Ola mouse. J ournal of Neurochemistry, 62: 24722475.

15. George EB, Glass J D \& Griffin J W (1995). Axotomy-induced axonal degeneration is mediated by calcium influx through ionspecific channels. Journal of Neuroscience, 15: 6445-6452.

16. Martinez AMB \& Ribeiro LCV (1998). UI- trastructural localization of calcium in peripheral nerve fibers undergoing Wallerian degeneration: an oxalate-pyroantimonate and X-ray microanalysis study. J ournal of Submicroscopic Cytology and Pathology, 30: 451-458.

17. Bartus RT (1997). The calpain hypothesis of neurodegeneration: evidence for a common cytotoxic pathway. Neuroscientist, 3: 314-327.

18. Banik NL (1992). Pathogenesis of myelin breakdown in demyelinating diseases: role of proteolytic enzymes. Critical Review on Neurobiology, 6: 257-271.

19. Waxman SG, Black JÁ, Ransom BR \& Stys PK (1993). Protection of the axonal cytoskeleton in anoxic optic nerve by decreased extracellular calcium. Brain Research, 614: 137-145.

20. Waxman SG, Black J Á, Stys PK \& Ransom BR (1992). Ultrastructural concomitants of anoxic injury and early post-anoxic recovery in rat optic nerve. Brain Research, 574: 105-119. 